\title{
Seminare / Séminaires / Seminari 2010
}

\section{Praxiseröffnung/-übernahme}

Themen

Juristische Aspekte (Praxisbewilligung, Zulassung zur Sozialversicherung, Vertragswesen), Gesellschaftsformen / Ehe- und Erbrecht (Trennung Privat- vom Geschäftsvermögen, Ehegüterstand, Erbschaftsplanung), Praxiseinrichtung (Inneneinrichtung, Kostenberechnung), Praxisadministration (Leistungserfassungs- und Abrechnungssysteme), Unternehmensbewertung einer Arztpraxis (Berechnung und Beurteilung des Unternehmenswertes), Finanzierung der Arztpraxis (Businessplan, Kredite, Absicherungsmöglichkeiten), Versicherungen/Vorsorge/Vermögen (Personen- und Sachversicherungen, Vorsorgeplanung).

\section{Sponsoren}

Die Kosten werden durch diverse Sponsoren (siehe www.fmhservices.ch) gedeckt.

\section{Daten}

$\begin{array}{llll}\text { K03 } & \begin{array}{l}\text { Donnerstag, 10. Juni } 2010 \\ \text { Bern }\end{array} & \text { Schmiedstube } \\ \text { 904-16.30 Uhr } & & \\ \text { K04 } & \begin{array}{l}\text { Donnerstag, 2. September 2010 } \\ \text { Zürich }\end{array} & \text { FMT } \\ & 9.00-16.30 \mathrm{Uhr} & \end{array}$

\section{Praxisübergabe}

\section{Themen}

Juristische Aspekte (Praxisübergabevertrag, allg. Vertragswesen, Übergabe der Krankengeschichten), Unternehmensbewertung einer Arztpraxis (Berechnung Inventarwert und Goodwill als Verhandlungsbasis), Versicherungen/Vorsorge/Vermögen (Übergabe/Auflösung von Versicherungsverträgen, Pensionsund Finanzplanung), Steuern (Steueraspekte bei der Praxisübergabe, Optimierung der steuerlichen Auswirkungen, Liquidations- und Grundstückgewinnsteuer, Bestimmung des optimalen Übergabezeitpunktes).

\section{Sponsoren}

Die Kosten werden durch diverse Sponsoren (siehe www.fmhservices.ch) gedeckt.

\begin{tabular}{lll} 
Daten & & \\
K08 & $\begin{array}{l}\text { Donnerstag, 17. Juni } 2010 \\
\text { Bern }\end{array}$ & 13.30-18.00 Uhr \\
K09 & $\begin{array}{l}\text { Donnerstag, 9. September 2010 } \\
\text { Zürich }\end{array}$ & FMT \\
\hline
\end{tabular}

\section{Praxiscomputerworkshop}

\section{Inhalt}

Die Workshopteilnehmer/innen erhalten im 1. Teil eine Einführung in die Anforderungen an ein Praxis- informationssystem. Anhand einer modernen vernetzten Praxisinfrastruktur werden die Beurteilungskriterien für eine praxis- und zukunftstaugliche Softwarelösung dargestellt. Checklisten sollen die schnelle Orientierung unterstützen und bei der Beurteilung und Wahl des Produkts konkrete Hilfe bieten. In Zusammenarbeit mit SGAM.Informatics werden die zentralen Elemente der elektronischen Krankengeschichte aufgezeigt. Ein Erfahrungsbericht eines EDV-Anwenders (Arzt) rundet den 1. Teil ab. Der 2. Teil umfasst die Präsentation von sechs Praxisadministrationssoftwarelösungen (Leistungserfassung, elektronisches Abrechnen unter Einbezug der TrustCenter, Agendaführung, Statistiken, Laborgeräteeinbindung, elektronische Krankengeschichte, Finanzbuchhaltungslösungen usw.).

\section{Ziel}

Die Teilnehmer/innen erhalten einen Anforderungskatalog, welcher ihnen erlaubt, ihre Vorstellungen für ein modernes Praxisinformationssystem besser zu formulieren und diese dem Softwarehersteller zu dessen Vorbereitung zu kommunizieren. Zudem erhalten sie einen ersten Überblick über führende Softwarelösungen.

\section{Daten}

\begin{tabular}{|llll}
\hline K14 & $\begin{array}{l}\text { Donnerstag, 24. Juni } 2010 \\
\text { Bern }\end{array}$ & 9.30-16.30 Uhr & BEA \\
Bern Expo
\end{tabular}

\section{Röntgen in der Arztpraxis}

Das Seminar richtet sich an Ärztinnen und Ärzte mit bestehender Praxis und an solche, die vor einer Praxiseröffnung oder Praxisübernahme stehen. Das Seminar ist auf maximal 15 Teilnehmer/innen beschränkt.

\section{Themen}

Vom konventionellen zum digitalen Röntgen, Rentabilität Röntgen in der Arztpraxis, Neue Vorschriften seit 1. Januar 2008, Evaluation und Beschaffung neuer oder gebrauchter Anlagen, Komplette Marktübersicht mit Preisen und Leistungskomponenten. Die Seminarteilnehmer erstellen und bearbeiten innerhalb des Seminars digitale Röntgenbilder und erhalten eine komplexe Dokumentation über alle Themen ein Vademekum rund ums Röntgen.

\section{Kosten}

Fr. 300.- (inkl. sämtlicher Kursunterlagen und Verpflegung). 
Daten

K16 Donnerstag, 26. August 2010 Niederscherli $9.30-16.00 \mathrm{Uhr}$

WIROMA AG

Ouverture et reprise d'un cabinet médical

\section{Contenu}

Business plan (préparation du plan de financement et crédit d'exploitation, financement par la banque), Aménagement (implantation, projet et concept d'aménagement, choix du mobilier, budget), Estimation d'un cabinet (inventaire et goodwill), Administration d'un cabinet médical (dans le cabinet, par la banque), Assurances (toutes les assurances à l'intérieur et autour du cabinet), Passage du statut de salarié à celui d'indépendant et fiscalité.

\section{Sponsors}

Les coûts sont pris en charge par divers sponsors (voir www.fmhservices.ch).

\section{Dates}

$\begin{array}{lll}\text { K21 } & \begin{array}{l}\text { Jeudi } 10 \text { juin } 2010 \\ \text { Neuchâtel } 17.00-21.30 \mathrm{~h}\end{array} & \text { La Maladière } \\ \text { K22 } & \begin{array}{l}\text { Jeudi } 2 \text { septembre } 2010 \\ \text { Genève } \quad 13.30-18.00 \mathrm{~h}\end{array} & \begin{array}{l}\text { Ramada } \\ \text { Park Hôtel }\end{array}\end{array}$

\section{Apertura e rilevamento di uno studio medico}

\section{Contenuto}

Business plan (preparazione del piano di finanziamento e del credito d'esercizio, prestito bancario), Pianificazione (insediamento, progetto e pianificazione, scelta del mobilio, budget), Valutazione di uno studio medico (inventario e goodwill), Amministrazione di uno studio medico (interna allo studio, rapporti con la banca), Assicurazioni (tutte le assicurazioni necessarie interne ed esterne allo studio), Passaggio dallo stato di dipendente a quello di indipendente, Fiscalità.

\section{Sponsor}

Diversi sponsor si fanno carico delle spese (si rimanda al sito www.fmhservices.ch).

\section{Date}

\begin{tabular}{|lll} 
K51 & $\begin{array}{l}\text { Mercoledì } 26 \text { maggio } 2010 \\
\text { Chiasso dalle } 14.00 \text { alle } 18.00\end{array}$ & $\begin{array}{l}\text { FMH } \\
\text { Fiduciaria } \\
\text { Services }\end{array}$ \\
K52 & $\begin{array}{l}\text { Martedì } 19 \text { ottobre } 2010 \\
\text { Chiasso dalle } 17.00 \text { alle } 21.00\end{array}$ & $\begin{array}{l}\text { FMH } \\
\text { Fiduciaria } \\
\text { Services }\end{array}$
\end{tabular}

\section{Anmeldung und Auskunft / Inscription et information / Iscrizioni e informazioni}

www.fmhservices.ch oder FMH Consulting Services, Sandra Stadelmann, Burghöhe 1, 6208 Oberkirch, Tel. 04192500 77, Fax 0419210586.

\section{Hinweis / Remarque / Osservazioni}

Bei sämtlichen Seminaren, bei denen die Kosten teilweise oder gänzlich von Seminarsponsoren gedeckt werden, werden die Teilnehmeradressen den jeweiligen Sponsoren zur Verfügung gestellt.

Les adresses des participants aux séminaires dont les coûts sont couverts en partie ou totalement par des sponsors sont communiquées aux sponsors concernés. Gli indirizzi dei partecipanti ai seminari, i cui costi sono coperti in parte o completamente da degli sponsor, vengono comunicati agli sponsor interessati.

\section{Annullierungsbedingungen / \\ Conditions d'annulation /}

\section{Condizioni d'annullamento}

Bei Abmeldungen oder Fernbleiben werden folgende Unkostenbeiträge erhoben:

Un montant est perçu pour une absence ou une annulation. Il est de:

Un importo verrà rimborsato in caso di assenza o annullamento. Esso sarà di:

- Fr. 50.- pro Person ab 14 Tagen vor Seminarbeginn / par personne dans les 15 jours avant / per persona entro i 15 giorni prima dell'inizio del seminario;

- Fr. 100.- pro Person ab 7 Tage vor Seminarbeginn oder Fernbleiben / par personne dans les 7 jours avant le début du séminaire / per persona entro i 7 giorni prima dell'inizio del seminario. 